\title{
Pre-, intra- and postoperative sonography of the abdominal wall in patients with incisional hernias repaired via a three-layered operative suture method
}

Dennis den Hartog, MD, ${ }^{1}$ Alfons H. M. Dur, MD, ${ }^{2}$ Alfons G. A. Kamphuis, MD, ${ }^{3}$

Wim E. Tuinebreijer, MD, PhD, MSc, MA,${ }^{2}$ John J. Hermans, MD,${ }^{4}$ Robert W. Kreis, MD, $\mathrm{PhD}^{2}$

${ }^{1}$ Department of Surgery, Erasmus MC, University Medical Center Rotterdam, Gravendijkwal 230, Office H-960, 3000 CA Rotterdam, the Netherlands

${ }^{2}$ Department of Surgery, Red Cross Hospital, Vondellaan 13, 1942 LE Beverwijk, the

Netherlands

${ }^{3}$ Department of Radiology, Red Cross Hospital, Vondellaan 13, 1942 LE Beverwijk, the

Netherlands

${ }^{4}$ Department of Radiology, Erasmus MC, University Medical Center Rotterdam,

Gravendijkwal 230, Office H-960, 3000 CA Rotterdam, the Netherlands 


\begin{abstract}
We illustrate the various sonographic (US) appearances of the abdominal wall following this type of repair, including partial and complete recurrences. Correlation is made with CT imaging. The three-layered anatomical reconstruction of an incisional hernia is described.
\end{abstract}




\section{Introduction}

Incisional hernias, which are hernias that occur through a surgical scar in the anterior abdominal wall, are serious complications of abdominal surgery. Incisional hernias occur in $11 \%-23 \%$ of laparotomies ${ }^{1}$ and can lead to serious morbidity from strangulation or

incarceration in $6 \%-14.6 \%$ of cases. ${ }^{2}$ Frequently, their diagnosis can be made through clinical examination, but small hernias and hernias in obese patients can be difficult to diagnose, which makes sonography (US) or CT imaging critical. The recurrence rate of incisional hernias after open suture repair may be as high as $54 \%,{ }^{3}$ and for open mesh repair specifically, recurrence rates can be up to $32 \% .{ }^{4}$ To decrease the recurrence rate, we developed a method that combines three-layered closure repair with extensive adhesiolysis. Using this repair method without mesh, we achieved recurrence rates that are comparable with the mesh procedure.

To determine the feasibility of this operation, however, it is important to examine and assess the quality and anatomic position of each abdominal wall layer. Five patients from our surgical department were selected between November 2006 and January 2008 for this pictorial essay. All US examinations were performed with an Aplio XG, model SSA-796A scanner (Toshiba Medical Systems, Tokyo, Japan) and 5-12 MHz PLT-1204AX linear transducer. CT examinations were performed with an Asteion 4-slice helical scanner (Toshiba Medical Systems) with typical acquisition protocols and sagittal and coronal reconstructions. The aim of this pictorial essay is to describe US anatomy of the abdominal wall before, during and after the three-layered anatomic reconstruction of an incisional hernia.

\section{NORMAL ANATOMY OF THE ABDOMINAL WALL}

The normal anatomy of the abdominal wall is shown a 55-year-old male who had not previously undergone abdominal surgery and was admitted for resection of a colonic carcinoma. He underwent preoperative CT and US examinations of the abdominal wall (Figure 1). 

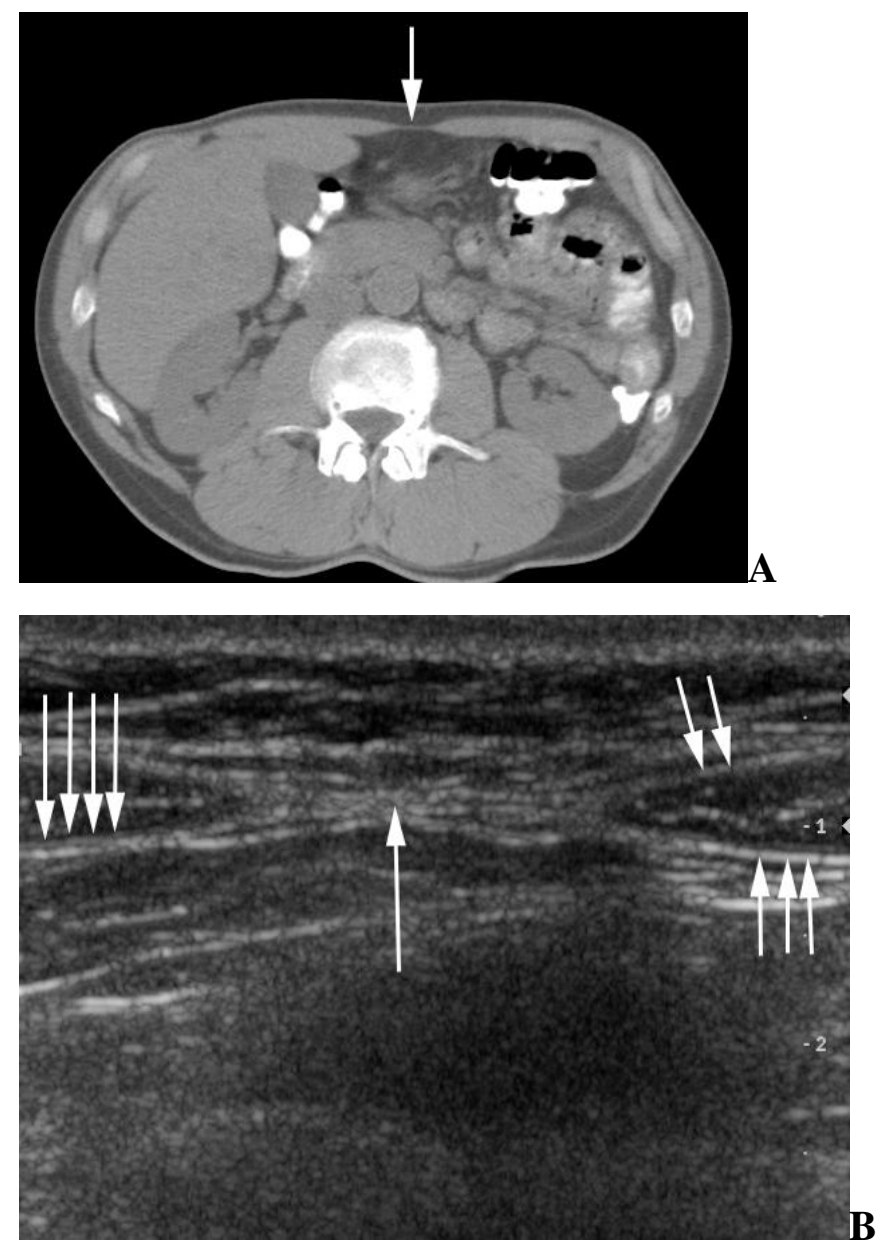

Figure 1 (A) Preoperative CT scan of a 55-year-old male shows the anatomy of the ventral abdominal wall and the linea alba (arrow) on the midline. On both sides of the linea alba are the rectus muscles. (B) Preoperative transverse sonogram shows the linea alba (arrow) on the midline. On both sides of the linea alba are the rectus muscles with anterior rectus fascia (two arrows). The peritoneum is marked with triple arrows, and immediately in front of the peritoneum is the posterior rectus fascia, a thinner line marked with four arrows.

The abdominal wall was opened through a midline incision. After opening the abdominal cavity, intraoperative US (IOUS) was performed to examine the abdominal wall adjacent to the incision. The median portion of the ventral abdominal wall consists of the two rectus sheaths. Each rectus sheath includes a rectus muscle between the anterior and posterior rectus fasciae, which join with the other side at the median line to form the linea alba. IOUS could differentiate between the peritoneum and the posterior rectus fascia after opening the rectus sheath and placing a forceps between the peritoneum and the rectus sheath (Figure 2). 

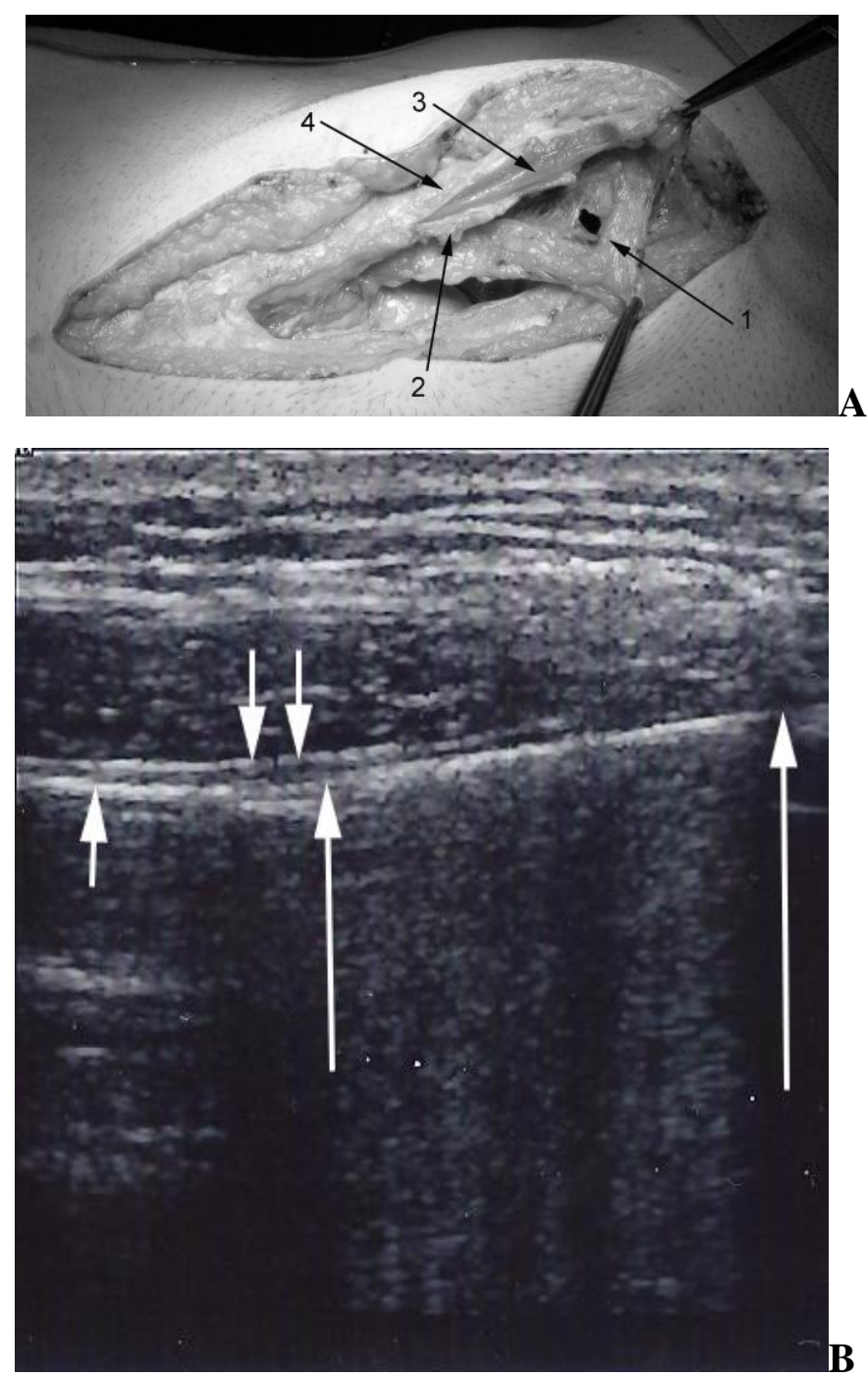

Figure 2 (A) Intraoperative photograph shows the anatomy of the abdominal wall through a midline incision. The lower forceps are holding the peritoneum with preperitoneal fat (arrow 1), which is separate from the posterior rectus fascia (arrow 2). The opened rectus sheath shows the rectus muscle (arrow 3 ) and the anterior rectus fascia (arrow 4) towards patient's head. (B) Intraoperative sonogram. The transducer is placed on the skin, in a transverse position to the right of the midline incision. The rectus sheath has been cut, and forceps (straight white line between the two long arrows) is shown between the posterior rectus fascia (two small arrows) and the peritoneum (one small arrow). The forceps was used to ensure that the anterior interface was the rectus fascia and the posterior one was the peritoneum.

US and CT were repeated postoperatively to show the healed median incision (Figure 3). After a midline incision, the linea alba usually heals, and the 2 rectus muscles remain separated. 

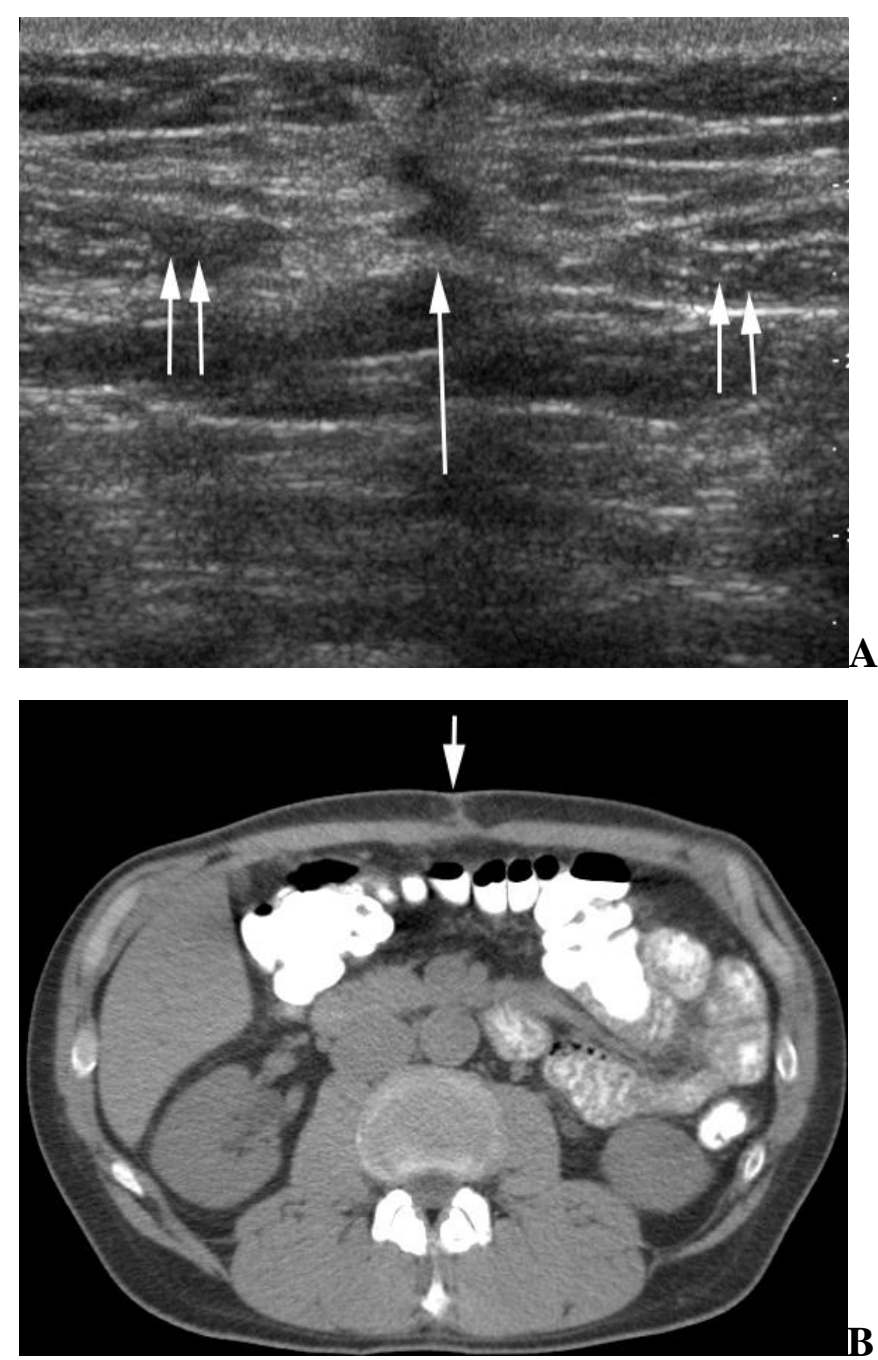

Figure 3 (A) Postoperative sonogram shows the anatomy of the midline abdominal wall after closure, the healing of a median incision, the healed linea alba (one arrow) within the midline, and scar tissue above the fascia of the linea alba. On both sides of the linea alba are the rectus muscles (double arrow) with their anterior and posterior rectus fasciae. (B) Postoperative CT shows the anatomy of the ventral abdominal wall after closure and healing of a median incision, the healed linea alba within the midline, and scar tissue (arrow) above the fascia of the linea alba.

\section{INCISIONAL HERNIA AFTER MEDIAN INCISION}

Incisional hernia after a median incision is shown in a 67-year-old male who had developed an incisional hernia after undergoing aortic-iliac reconstruction for arterial occlusive disease 5 years earlier (Figure 4). 


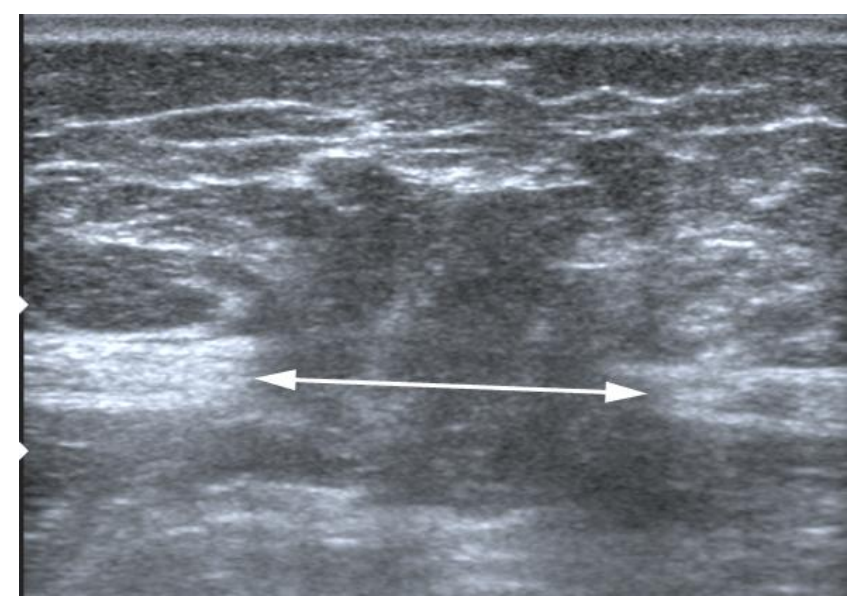

Figure 4. Preoperative transverse sonogram of an incisional hernia after reconstruction of an aortic-iliac occlusion that occurred 5 years previously shows a defect of the linea alba (double arrow) with a protrusion of the hernial sac consisting of preperitoneal fat.

The progressive enlargement of the hernial sac shifts the rectus muscles laterally. During realtime US examination, the protrusion of the hernial sac can be augmented when the patient performs a Valsalva maneuver.

\section{THREE-LAYERED DIRECT SUTURE REPAIR OF INCISIONAL HERNIA AFTER} MEDIAN INCISION

Our three-layered closure repair technique for incisional hernias consists of dissecting the retracted midline edges of the anterior and posterior sheath of the rectus abdominis muscle after extensive adhesiolysis. The peritoneum and posterior sheath are closed together in the first layer. The rectus abdominis muscles are placed in contact in the second layer, and the anterior sheath is closed in the third layer (Figure 5).

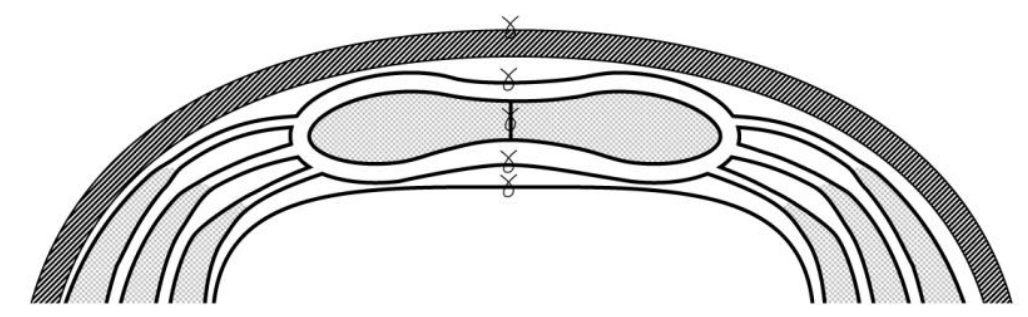

Figure 5. Diagram showing the 3-layered closure technique. The anterior/posterior rectus fasciae and the rectus muscles are sutured in separate layers, forming the three layers of the repair. In reality, the peritoneum, posterior rectus fascia and pieces of the rectus muscle are 
sutured together continuously, and the anterior rectus fascia and pieces of rectus muscle are also sutured continuously. This type of closure prevents the muscle from tearing. Thus, the three-layered closure consists of the apposition of three layers with two sutured layers.

The 2nd phase of this reconstruction consists of suturing the two rectus muscles together along the median line by including pieces of rectus muscle $1 / 2$ centimeter from the median edge and including the anterior or the posterior fascia. On US, the linea alba has disappeared and the two rectus muscles are in continuity on the median line (Figure 6).

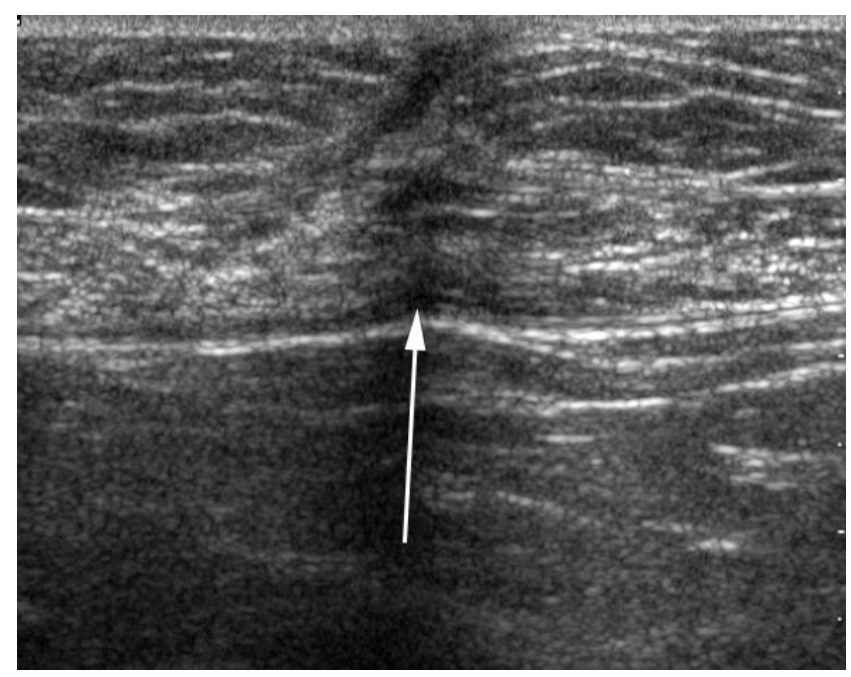

Figure 6. Postoperative transverse sonogram 2 years following the three-layered direct suture repair of an incisional hernia. The rectus muscles are attached to each other, and the linea alba has disappeared. Scar tissue (arrow) separates the rectus muscles. The posterior fasciae of both rectus muscles are continuous.

\section{RECURRENCE AFTER THREE-LAYERED DIRECT SUTURE REPAIR OF AN INCISIONAL HERNIA FOLLOWING MEDIAN INCISION}

US appearances of recurrent incisional hernias after the three-layered direct suture repair of a hernia through a median incision are shown in Figures 7 and 8. Recurrences can be partial and total (Figure 7). There is complete breach through the three sutured layers with possible protrusion of a hernial sac. 


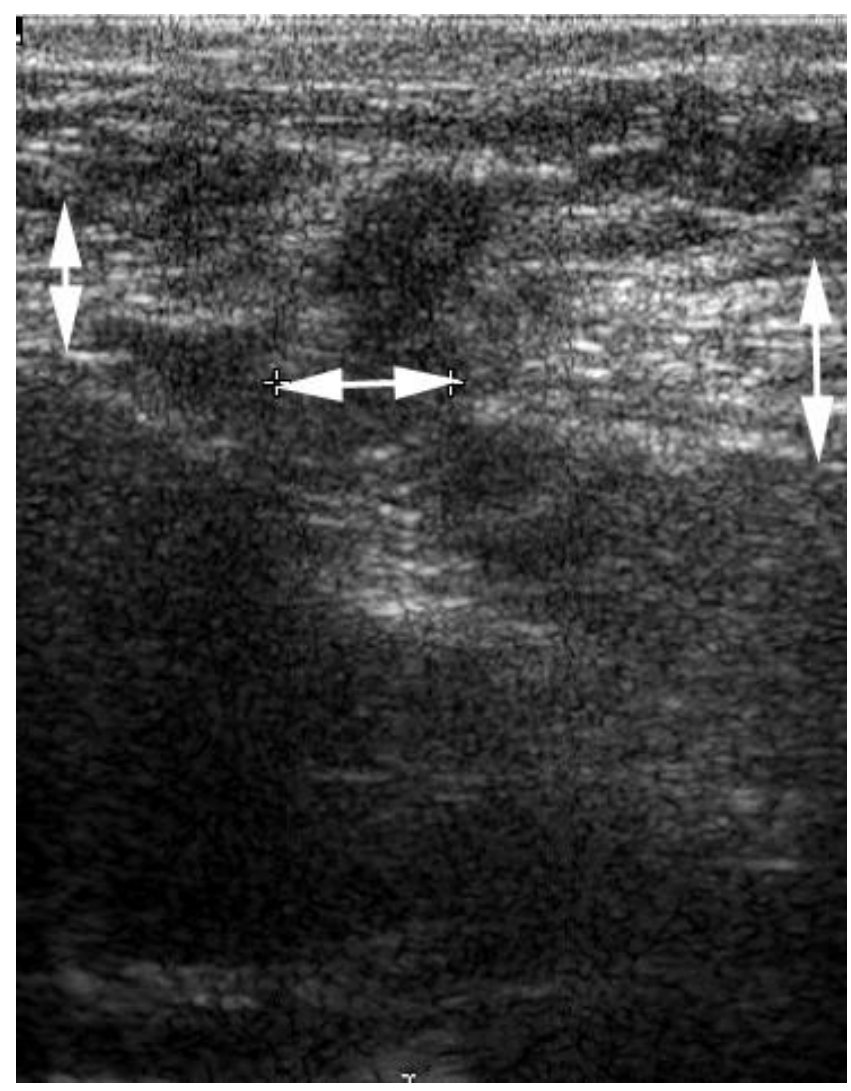

Figure 7. Complete recurrence 2 years after a three-layered direct suture repair of an incisional hernia. Transverse sonogram shows a complete discontinuity (horizontal double arrow) between the two rectus muscles (vertical double arrows) with protrusion of a small hernial sac.

A partial recurrence is defined as a defect of only the posterior fascia of the rectus sheath with an intact anterior fascia (Figure 8). In a partial defect, the outer layer of the repair (anterior rectus fascia) remains intact. 


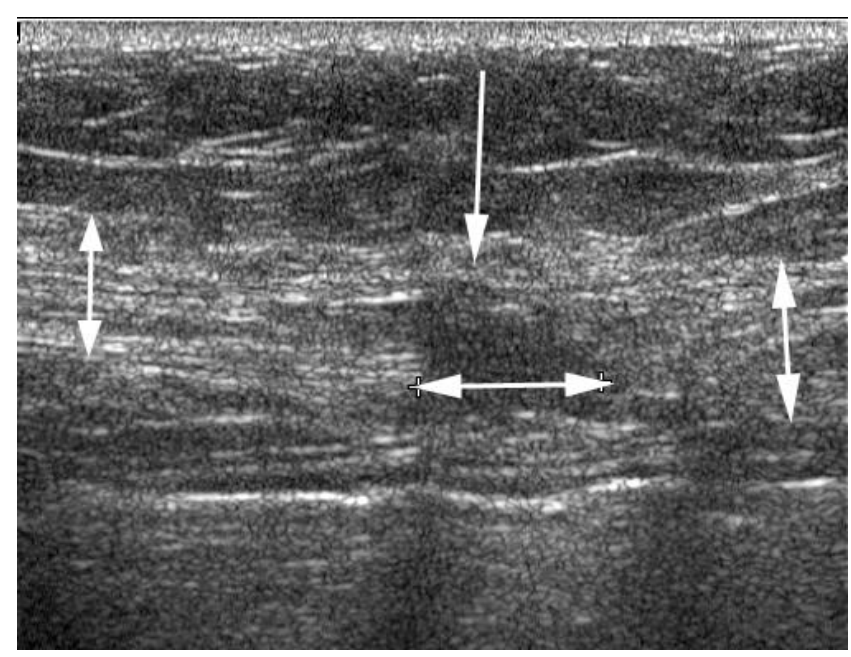

Figure 8. Partial recurrence of an incisional hernia 2 years after a three-layered direct suture repair. Transverse midline sonogram shows the partial defect (horizontal double arrow) and no protrusion of a hernial sac. The anterior rectus fascia is intact (vertical arrow). Vertical double arrows indicate the rectus muscles.

\section{Discussion}

Repairs of incisional hernias can be performed using either open or laparoscopic techniques. ${ }^{5}$ Open techniques include a simple hernioplasty (Mayo duplication or fascia-adaptation), component separation, or mesh repair. The recurrence rate of incisional hernias after open suture repair may be as high as $54 \%,{ }^{3}$ and for open mesh repair specifically, recurrence rates can be up to $32 \% .{ }^{4}$ However, the infection rate is higher in patients with open mesh repair and was, for instance, $10.1 \%$ in a Cochrane review, which pooled data from 2 studies. $^{6}$ Recurrence rates for laparoscopic repair are comparable with those obtained with the open mesh procedure but offer a shorter hospital stay. ${ }^{1}$ High incidence rates of incisional and recurrent incisional hernias make imaging modality very important for this condition. Whereas the CT features of incisional hernias have been clearly described, ${ }^{7-9}$ the US characteristics of ventral hernias have not been reported in detail. ${ }^{10,11}$ An observational study compared US with CT in the diagnosis of incisional hernias, ${ }^{12}$ but in general, the previous studies did not focus on the abdominal wall layers following the repair of an incisional hernia. This type of description is necessary to evaluate the concept of closure across all three layers, including the anterior fascia, the rectus abdominal muscles and the posterior fascia. 


\section{References}

1. Cassar K, Munro A. Surgical treatment of incisional hernia. Br J Surg 2002;89(5):534.

2. Nieuwenhuizen J, Halm JA, Jeekel J, et al. Natural course of incisional hernia and indications for repair. Scand J Surg 2007;96(4):293.

3. Paul A, Korenkov M, Peters S, et al. Unacceptable results of the Mayo procedure for repair of abdominal incisional hernias. Eur J Surg 1998;164(5):361.

4. Burger JW, Luijendijk RW, Hop WC, et al. Long-term follow-up of a randomized controlled trial of suture versus mesh repair of incisional hernia. Ann Surg 2004;240(4):578.

5. Korenkov M, Paul A, Sauerland S, et al. Classification and surgical treatment of incisional hernia. Results of an experts' meeting. Langenbecks Arch Surg 2001;386(1):65.

6. den Hartog D, Dur AHM, Tuinebreijer WE, et al. Open surgical procedures for incisional hernias. Cochrane Database of Systematic Reviews 2008;3.

7. Ishida H, Konno K, Hamashima Y, et al. Anterior abdominal wall pathologies detected by high-frequency annular array. Eur J Ultrasound 1998;7(3):167.

8. Aguirre DA, Santosa AC, Casola G, et al. Abdominal wall hernias: imaging features, complications, and diagnostic pitfalls at multi-detector row CT. Radiographics 2005;25(6):1501.

9. Harrison LA, Keesling CA, Martin NL, et al. Abdominal wall hernias: review of herniography and correlation with cross-sectional imaging. Radiographics $1995 ; 15(2): 315$.

10. Deitch EA, Engel JM. Ultrasonic diagnosis of surgical diseases of the anterior abdominal wall. Surg Gynecol Obstet 1980;151(4):484.

11. Young J, Gilbert AI, Graham MF. The use of ultrasound in the diagnosis of abdominal wall hernias. Hernia 2007;11(4):347.

12. Yeh HC, Lehr-Janus C, Cohen BA, et al. Ultrasonography and CT of abdominal and inguinal hernias. J Clin Ultrasound 1984;12(8):479. 